\title{
IDENTIFICATION OF ACID MUCOPOLYSACCHARIDES BY PAPER CHROMATOGRAPHY *
}

\author{
C. WILLIAM CASTOR" AND EMILY L. DORSIEWITZ \\ Department oj Internal MIedicine, \\ The University of Michigan Medical Conter and the Rachham Arthritis Research Unit, \\ Ann Arbor, Mich. (U.S.A.)
}

(Received April roth, $\left.19 G_{3}\right)$

Acid mucopolysaccharides, a family of carbohydrate macromolecules found in connective tissue "ground substance", have been isolated and studied by several investigators ${ }^{1-3}$. Isolation techniques applicable to large masses of tissue yield sufficient purified acid mucopolysaccharide to permit accurate identification by chemical analysis. However, even tentative identification of these substances in plasma, urine, or tissue culture media may be difficult due to low concentrations and limited total sample. Methods are available for the quantitative measurement of acid mucopolysaccharides in such limited samples ${ }^{4-6}$. Although progress in qualitative identification of specific acid mucopolysaccharides in small samples is less satisfactory, chromatographic methods appear promising as a means of rapid, tentative identification of material available in amounts inadequate for exhaustive chemical analysis.

Solvent systems employing phosphate buffer in propanol or ethanol have been used to demonstrate metachromatic material in urine and plasma possessing chromatographic mobility resembling chondroitin sulfate ${ }^{4,7}$. While these systems tend to distinguish heparin from chondroitin sulfate (cartilage origin), hyaluronate always remained at the origin, making its identification doubtful by these methods. A solvent system using dilute ammonium formate in isopropanol has been devised which will completely resolve chondroitin sulfate and heparin ${ }^{8}$. In addition, a multiple solvent system employing silicated glass paper appeared to separate most of the known mammalian acid mucopolysaccharides ${ }^{9}$. This technique offers the advantage of speed of execution but seems useful only with highly purified mucopolysaccharide samples.

In view of the limited methodology available for chromatographic identification of acid mucopolysaccharides, we have developed a system which distinguishes the known mammalian mucopolysaccharides and offers several advantages: ( $I$ ) it is rapid and reproducible, (2) it distinguishes hyaluronate by actual movement of the compound, and (3) significant protein contamination of acid mucopolysaccharides does not interfere. On the basis of the methods described below it appears feasible to identify hyaluronic acid, heparin, heparin monosulfate, and chondroitin sulfates $A, B$ and $C$ with some certainty. Identification of keratosuliate is less certain by these methods

This investigation was supported by United States Public Health Service Grant A-3665.

* Research Career Development Awarclee, United States Public Health Service. 
MATERIALS AND METHODS

\section{Acid mucopolysaccharide reference compounds}

Hyaluronic acid and chondroitin sulfate $C$ were prepared from human umbilical cord by peptic digestion and alcohol fractionation ${ }^{10}$. Chondroitin sulfates $A$ and $B$ (human skin) and chondroitin sulfate $C$ (shark cartilage) were prepared by papain digestion and alcohol fractionation; the final products were isolated as the sodium salts from methanolic solutions of the respective mucopolysaccharide-cetylpyridinium complexes ${ }^{2}$. Chondroitin was prepared by desulfation of chondroitin sulfate $\mathrm{A}^{11}$. Heparin samples included products from the Organon, Upjohn, and Roussel Companies. Examples of chondroitin sulfates $A, B$, and $C$, heparitin monosulfate, and keratosulfate were available as gifts from Dr. J. A. CIFONEL,L, University of Chicago, and Dr. KARL MEYER, Columbia University. Hyaluronic acid prepared from human umbilical cord by electrodeposition was provided by Dr. SAUt Roseman, The University of Michigan ${ }^{12}$.

Oligosaccharides of hyaluronic acid and chondroitin sulfate $A$ were prepared by prolonged hydrolysis with testicular hyaluronidase followed by dialysis of the digest. The aqueous dialysis bath was passed through a column of Dowex 5o, $\mathrm{H}+$ resin and then concentrated by rotary evaporation and lyophilization.

\section{Procedure}

Ascending chromatography was carried out in II in. rectangular chromatography jars. Samples were spotted with micropipettes, using 20 to $40 \mu \mathrm{g}$ of acid mucopolysaccharide in 5 to Io $\lambda$ of diluent. Development times ranging from $\mathrm{I}-\mathrm{I} 2 \mathrm{~h}$ were explored. Two to three hours was usually adequate to elicit the maximal resolution for a particular system.

For chromatograms which were to be developed by the "Type I" solvent systems an extra step was interposed before development in the solvent. After the sample spot was dry, the paper was immersed for $3 \mathrm{~min}$ in o.I \% cetylpyridinium chloride in $0.004 M$ phosphate buffer, $\mathrm{pH} 7.0$, and then allowed to dry over night at room temperature. Chromatograms to be developed in "Type II" solvent systems may be developed as soon as the sample spots are dry. Schleicher and Schuell 589 blue ribbon paper proved satisfactory for all solvent systems.

\section{Solvent systems}

Type $I$. In this group of solvents we have utilized and extended the information originally developed by SCOTT ${ }^{2}$ regarding the solubility of various mucopolysaccharidecetylpyridinium (MPS-CPC) complexes in salt solutions of varying ionic strength. After treating the chromatogram with o.I\% cetylpyridinium chloride, as noted above, the acid mucopolysaccharides are "fixed" and will remain at the origin if the chromatogram is developed with distilled water. However, if a salt solution of sufficient ionic strength to dissolve the MPS-CPC complex is used to develop the chromatogram, the mucopolysaccharide will move with the solvent front. "Type I" solvents, then, identify the solubility characteristics of a particular MPS-CPC complex and the critical salt concentration is the one which will allow migration of the acid mucopolysaccharide to the solvent front. Lithium chloride, magnesium chloride, aluminum chloride and ferric chloride solutions were examined in some detail as solvents. 
After development of the chromatogram in one of these solvents, it is air dried and then placed in the $0 . I \%$ CPC solution for $3 \mathrm{~min}$, followed by $95 \%$ ethanol, $3 \mathrm{~min}$; absolute ethanol, $3 \mathrm{~min}$; acetone, $2 \mathrm{~min}$ and then stained.

$T y p e I I$. As noted earlier, several investigators have used solvent systems composed of a dilute buffer and an organic solvent for the partial identification of the sodium or potassium salts of mucopolysaccharides. After carefully examining the ammonium formate-isopropanol system described by SPOLTER AND MARx ${ }^{8}$, we ultimately settled on a minor modification which has reduced development time as its chief virtue. For routine purposes we used $0.04 M, \mathrm{pH} 3.5$ ammonium formate and methanol in a $45: 55$ ratio.

\section{Staining chromatograms}

Chromatograms are stained for $3 \mathrm{~min}$ in an Azure $A$ solution made up as follows: $200 \mathrm{mg}$ Azure $\mathrm{A}$ in a mixture of $25 \mathrm{ml}$ water, $200 \mathrm{ml}$ acetone and $750 \mathrm{ml}$ methanol.

After staining, the chromatogram may be differentiated in 3:I methanol-acetone until the background is adequately cleared, and then air dried.

\section{Type I solvents}

\section{RESULTS}

Magnesinm chloride. A solvent system employing magnesium chloride allowed division of the several reference compounds into three groups. Figs. I, 2, and 3 illustrate the solubilization and consequent mobility of different MPS-CPC complexes at three separate concentrations of magnesium chloride. Hyaluronate stains distinctly less metachromatically than the sulfated compounds, and the streaking is apparently

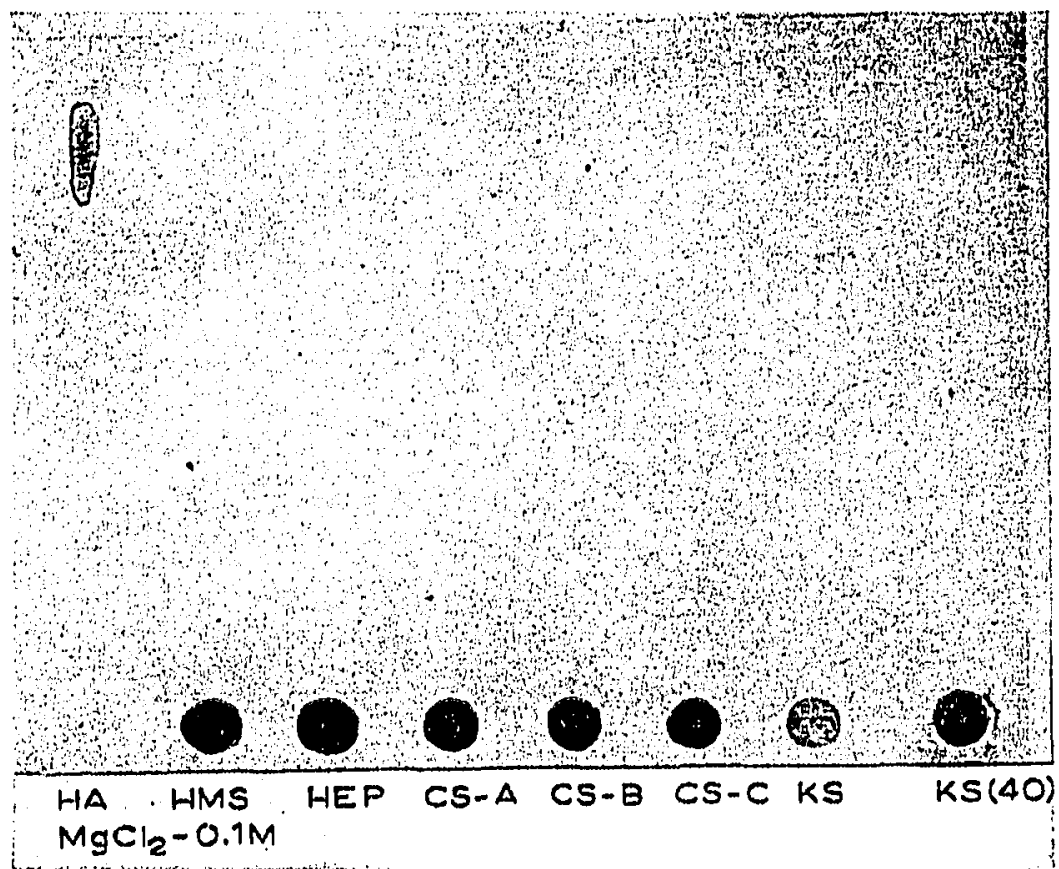

Fig. I. Chromatogram of acicl mucopolysaccharicles. HA $=$ hyaluronic acicl; IMS $=$ heparitin monosulfate; $\mathrm{HEP}=$ heparin; $\mathrm{CS}-\mathrm{A}=$ chondroitin sulfate $\mathrm{A} ; \mathrm{CS}-\mathrm{J}=$ chondroitin sulfate $\mathrm{B}$ (dermatan sulfate); $\mathrm{CS}-\mathrm{C}=$ chondroitin sulfate $\mathrm{C}$; and $\mathrm{KS}=$ keratosulfate. All applications were $5 \mu 1$ volumes containing $20 \mu \mathrm{g}$ of mucopolysaccharide cxcept $\mathrm{KS}(40)$ which represents $40 \mu \mathrm{l}$, or IGo $\mu \mathrm{g}$ of mucopolysaccharicle. 


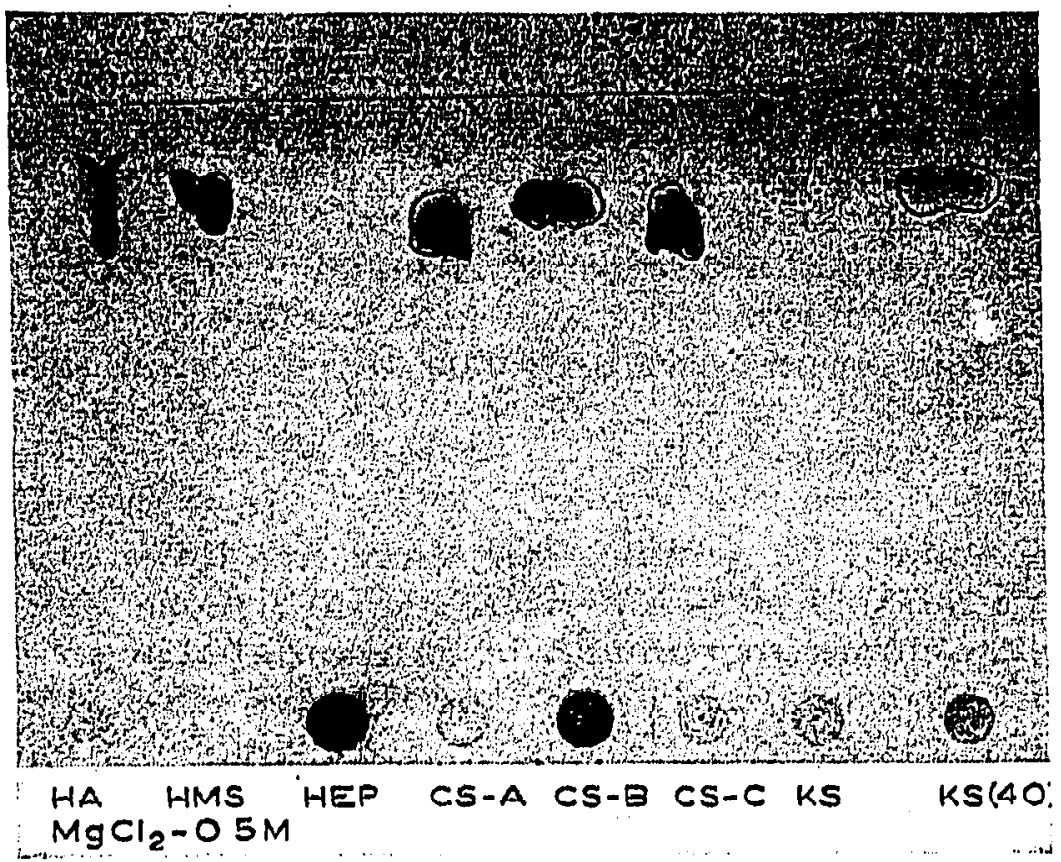

Fig. 2. Chromatogram of acid mucopolysaccharides. For abbreviations and conditions, see Fig. I.

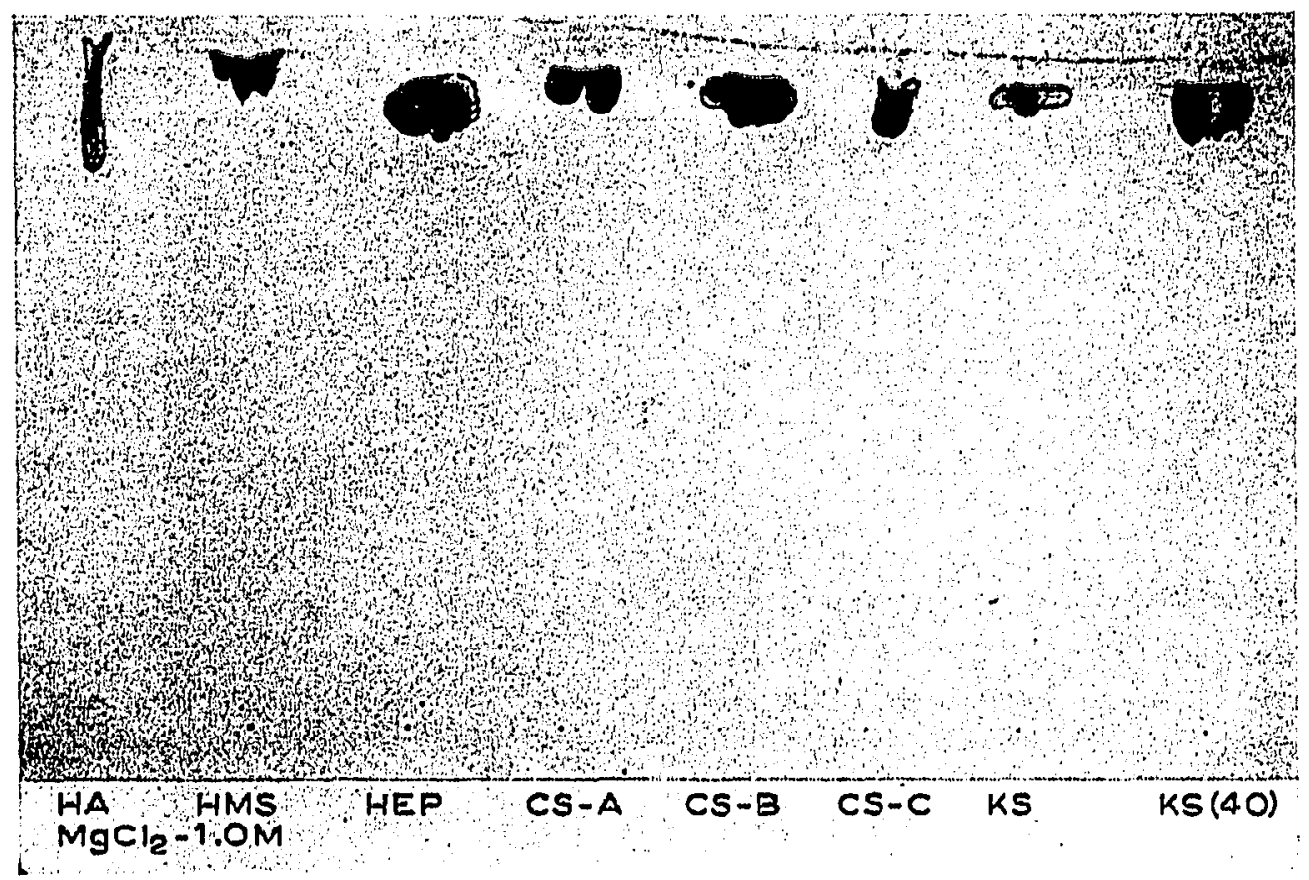

Fig. 3. Chromatogram of acid mucopolysaccharides. For abbreviations and conditions, see Fig. I. 
related to the very high molecular weight of viscous, relatively undegraded hyaluronate prepared by electrodeposition. Partially degraded hyaluronate (produced either by testicular hyaluronidase or by more drastic isolation methods) tends to move as a discrete spot near the front. Heparitin monosulfate and the three chondroitin sulfates stain with a similar deep blue metachromasia, while heparin has a reddish hue. Keratosulfate in equal concentration gave a pale metachromatic spot.

The concentrations of magnesium chloride indicated in Figs. I, 2, and 3 were chosen primarily because they provide clear cut distinction of hyaluronate and heparin from each other and from the remaining compounds. Actually, magnesium chloride concentrations ranging from $0.05 M$ to $0.25 M$ will solubilize the hyaluronate-CPC complex without moving the sulfated compounds. In the range from $0.3 M$ to $0.5 M$ $\mathrm{MgCl}_{2}$ the sulfated compounds other than heparin began to move with the solvent front. The possibility that the chondroitin sulfates, keratosulfate, and heparitin monosulfate might be separated by fine gradations in the ionic strength of the salt solution was examined. Magnesium chloride concentrations of $0.300 M, 0.330 M$ $0.350 M, 0.362 M, 0.375 M, 0.387 M$, and $0.400 M$ tended to move heparitin monosulfate, chondroitin sulfate $A$, chondroitin sulfate $C$ and chondroitin sulfate $B$ in that order as the salt concentration was increased. However, the overlap of mobilities was so great as to preclude practical application in identification of an unknown.

Aluminum chloride and ferric chloride. Chondroitin sulfates A, B, and C, keratosulfate and heparitin monosulfate are not resolved by the magnesium chloride system, and in an effort to resolve this quintet many other solvents were examined. Aluminum chloricle, $0.40 M$, proved useful since it moved four of these five to the solvent front, leaving chondroitin sulfate B conspicuously at the origin (see Fig. 4). Ferric chloride, $0.5 M$, will solubilize and move hyaluronate, keratosulfate and chondroitin sulfate A (Fig. 5). Heparitin monosulfate tends to move as a streak in this solvent. Of primary importance is the failure of chondroitin sulfates $\mathrm{B}$ and $\mathrm{C}$ to move.

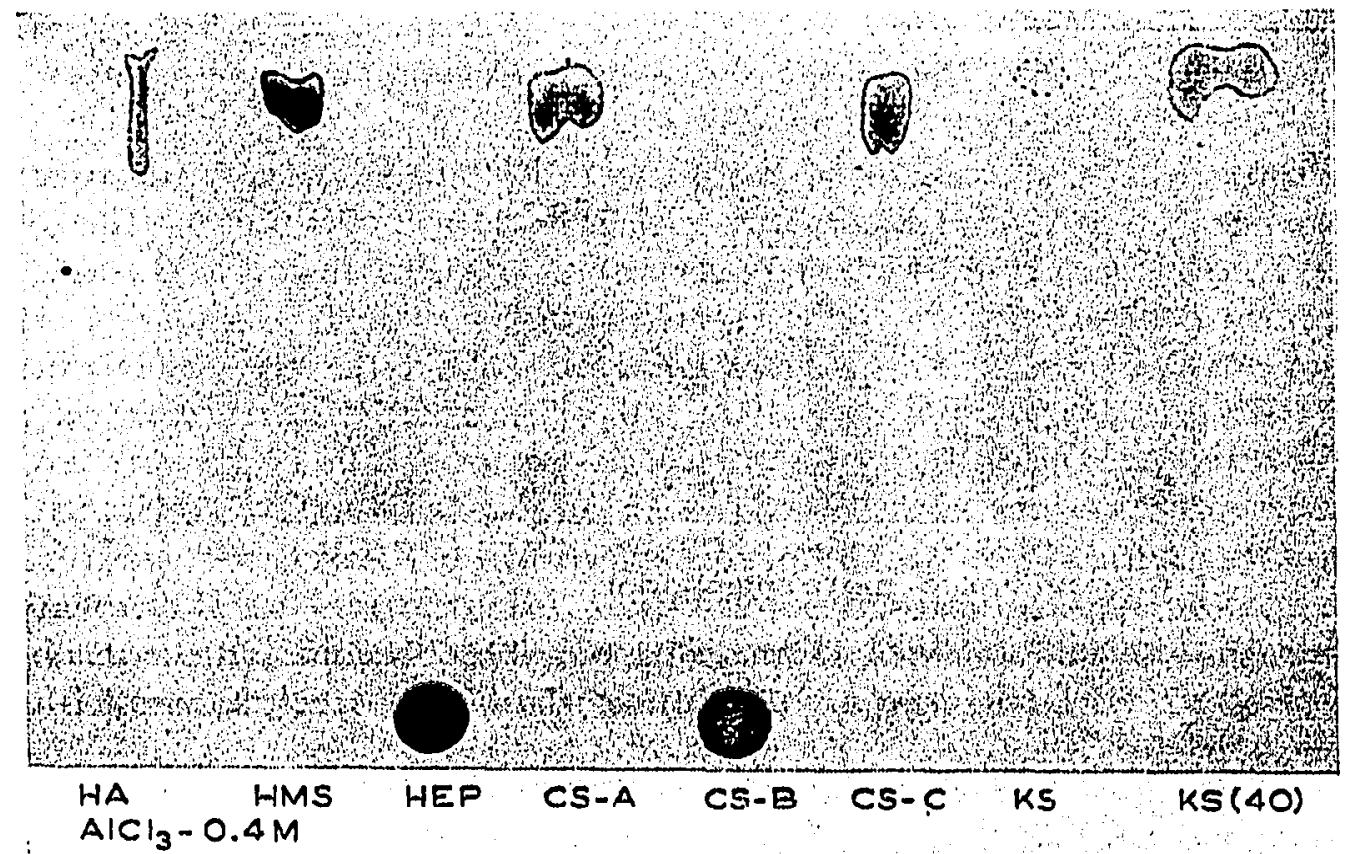

Fig. 4. Chromatogram of acid mucopolysaccharicles. For abbreviations and conditions, seo Fig. I. 


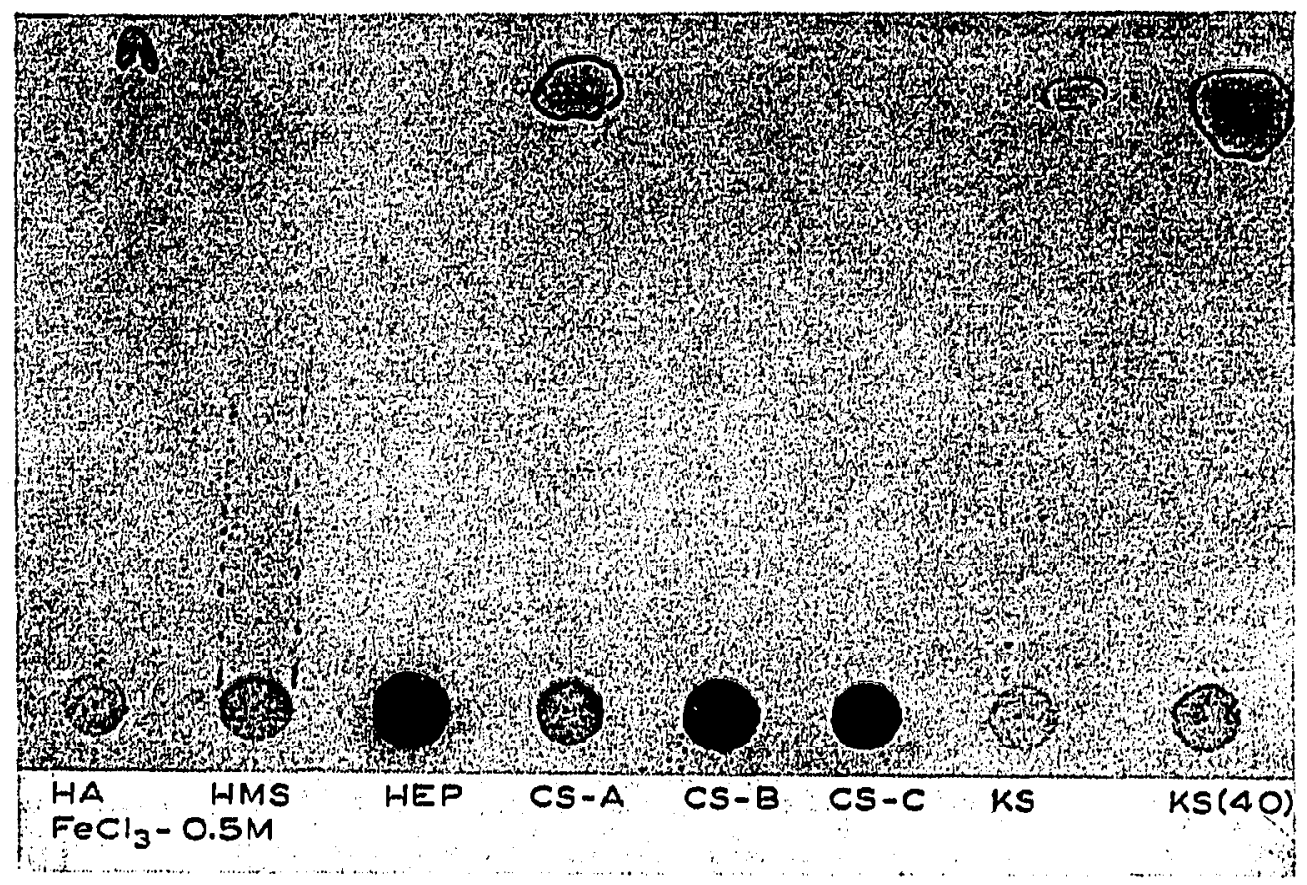

Fig. 5. Chromatogram of acid mucopolysaccharicles. For abbreviations and conditions, see Fig. I.

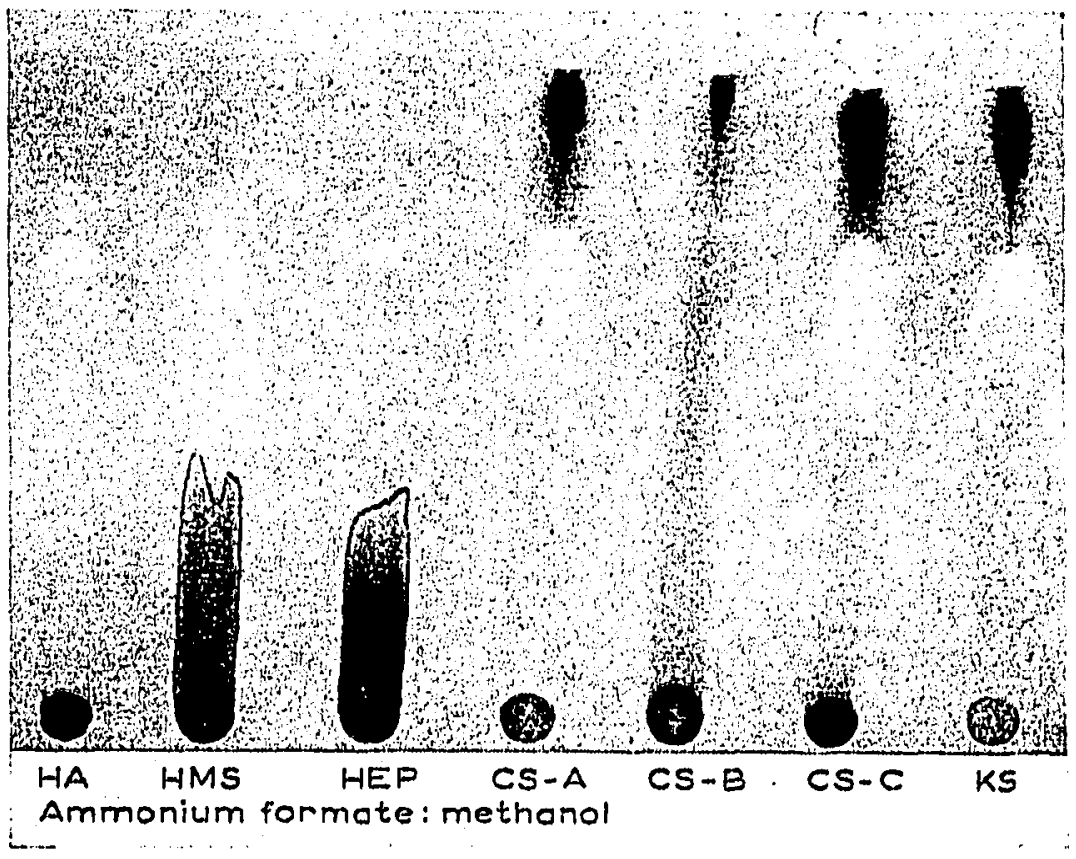

Fig. G. Chromatogram of acid mucopolysaccharides. For abbreviations and conditions, sce Fig. I. 


\section{Type II solvent systems}

The type II solvent system employed a dilute buffer in combination with one of the short chain alcohols. As Fig. 6 demonstrates, the Type II system also divides the mucopolysaccharide family into three distinct classes. At one extreme is hyaluronate which is essentially origin bound, and at the other extreme are the three chondroitin sulfates and keratosulfate which move with the solvent front. In between these extremes is the distinctive picture presented by heparin and heparitin monosulfate. These latter two substances exhibit a characteristic streak extending from the origin to a point about half way to the solvent front. Since heparin can be distinguished in the Type I system ( $1.0 M \mathrm{MgCl}_{2}$ ), it follows that an unknown which gave this streak effect in the Type II system and moved with $0.5 M \mathrm{MgCl}_{2}$ would be identified as heparitin monosulfate.

Table I summarizes the chromatographic behaviour of the various acid mucopolysaccharides. Distinctive patterns of behaviour occurred for five mucopolysaccharides, while chondroitin sulfate $A$ and keratosulfate showed many similar characteristics.

TABLE I

COMPARATIVE BEHAVIOUR OF ACID MUCOPOLYSACCHARIDIES IN DIFFERENT SOLVENT SYSTIEMS

\begin{tabular}{|c|c|c|c|c|c|c|}
\hline & $\stackrel{\circ}{\mathrm{O} g \mathrm{M}} \mathrm{M}$ & $\begin{array}{l}0.5 M \\
\mathrm{MgCl}_{\mathrm{g}}\end{array}$ & $\begin{array}{l}\mathrm{S} . \mathrm{OM} \\
\mathrm{M} \mathrm{MCl} l_{2}\end{array}$ & $\begin{array}{l}0.4 M \\
A I C L\end{array}$ & $\begin{array}{l}0.5 M \\
P_{L} C l_{\mathrm{s}}\end{array}$ & Type II \\
\hline Hyaluronic acid & $\mathrm{F}^{*}$ & $\mathrm{~F}$ & $\mathrm{~F}$ & $\mathrm{~F}$ & $\mathrm{~F}$ & $0^{\star \star}$ \\
\hline Keratosulfate & 0 & $\mathrm{~F}$ & $\mathrm{~F}$ & F & F & $\mathrm{F}$ \\
\hline Chondroitin sulfate A & 0 & $\mathrm{I}$ & $\mathrm{F}$ & $\mathbf{F}$ & $\mathrm{F}$ & $\mathrm{F}$ \\
\hline Chondroitin sulfate $B$ & 0 & $\mathrm{~F}$ & $F$ & $\mathrm{O}$ & 0 & $\mathrm{~F}$ \\
\hline Chondroitin sulfate $C$ & 0 & $\mathrm{~F}$ & $\mathrm{~F}$ & F & $\mathrm{O}$ & $\mathrm{F}$ \\
\hline I-Icparitin monosulfate & 0 & $\mathrm{~F}$ & $\mathrm{~F}$ & F & $S^{* * *}$ & $\mathbf{S}$ \\
\hline Heparin & 0 & 0 & $\vec{F}$ & 0 & $\mathrm{O}$ & $\mathbf{S}$ \\
\hline
\end{tabular}

\footnotetext{
" "IF" inclicates substance moves to front.

* " "O" inclicates substance is origin bound.

* * "S" inclicates substance streales from origin.
}

It is important, however, to note that while keratosulfate in $20 \mu \mathrm{g}$ amounts yielded spots of intensity equalling the chondroitin sulfates in the Type II systems, (Fig. 6), that this amount of keratosulfate is virtually not recorded in Type I systems. As seen in Figs. 2-5, eight times as much keratosulfate is required to produce spots of intensity comparable to other mucopolysaccharides. The precise reason for the apparent loss of keratosulfate from the Type I systems on reaching the critical salt concentration for the MPS-CPC complex is unclear. Fortunately, the absence of uronic acid in keratosulfate makes distinction from chondroitin sulfate $A$ possible by simple chemical analysis, even with a limited sample. From a practical standpoint, it is probably reasonable to characterize a substance as chondroitin sulfate $A$, if $20 \mu \mathrm{g}$ spots (based on uronic acid) behave according to the table. A mixture of chrondroitin sulfate $A$ and keratosulfate obviously could not be separated by these systems.

\section{The effect of several variables on the chromatographic systems}

Several different salts of chondroitin sulfate $A$ were prepared and rested in the Type II solvent system. Calcium, sodium, and ammonium salts behaved identically in this 
system. We have varied the $\mathrm{pH}$ of the ammonium formate-methanol system between 3.5 and 7.I without significant change in the chromatographic behaviour of the reference acid mucopolysaccharides. Pretreating the paper with a variety of substances, including sodjum tetraborate and sodium silicate did not increase the discriminatory capability of the Type II system. Increasing the ionic strength of the buffer in the Type II system resulted in decreased mobility. Dialyzable oligosaccharides from chondroitin sulfate $A$ migrated to the front in the ammonium formate-methanol system like the parent polymer. The oligosaccharide from hyaluronic acid also migrated with the front in this system, unlike the parent polymer which is bound to the origin. Neither oligosaccharide could be studied by the Type I system since the low molecular weight compounds, or their initial complex with CPC, are apparently dissolved in the original CPC bath and thus were lost from the paper.

The effect of protein contamination on the efficacy of these systems was tested for all the mucopolysaccharides. Each substance was dissolved in serum and then re-isolated by the euglobulin-heat precipitation method ${ }^{6}$. This final sample was dialyzed free of salt, lyophilized and solubilized in a small volume of water. These mucopolysaccharide samples contained up to $50 \%$ serum protein. The chromatographic behaviour of the serum protein-contaminated samples was identical to that shown by the protein-free acid mucopolysaccharides.

Monovalent salts, as lithium chloride, may be substituted for the three magnesium chloricle solvents. When this is done, O. $2 M$, I.o $M$, and $2.0 M$ lithium chloride solutions will yield the same data as the magnesium chloride solutions used here.

DISCUSSION

Resolution of mixtures of mucopolysaccharides may be undertaken to a limited extent by the methods outlined. Both heparin and hyaluronate may be identified in any mixture. Heparitin monosulfate may be distinguished when mixed with hyaluronate or heparin, but mixtures with the other sulfated compounds are not resolved with certainty. Further, mixtures of all the chondroitin sulfates and keratosulfate can not be completely resolved. In a mixture of chondroitin sulfates, one can identify chondroitin sulfate $A$, but can not distinguish between chondroitin sulfate $B$ alone and the combination of chondroitin sulfate $B$ and chondroitin sulfate $C$ as the other components of the mixture.

Chondroitin sulfate $\mathrm{C}$ isolated in this laboratory from shark fin cartilage did not behave chromatographically like chondroitin sulfate $\mathrm{C}$ of human origin. In general, it required higher salt concentrations to solubilize the MPS-CPC complex. This is consistent with the higher degree of sulfation found in shark cartilage chondroitin sulfate $\mathrm{C}^{13}$.

It should be recognized that in the chromatographic systems described in this report, that chondroitin (a desulfated chondroitin sulfate) is indistinguishable from hyaluronic acid. This material is only known to occur in the cornea, consequently confusion of chondroitin and hyaluronic acid is unlikely in most materials.

Mucopolysaccharide isolated from normal human urine was shown to consist primarily of chondroitin sulfate $A$ by application of these chromatographic methods. Examination of mucopolysaccharides isolated from pooled blood donor plasma revealed two components, one of which cluromatographed like chondroitin sulfate $A$. 
Chromatography of mucopolysaccharicles isolated from culture media which supported the growth of human connective tissue cells shows hyaluronic acid to be the predominant substance formed ${ }^{14}$.

Chromatographic identification of acid mucopolysaccharides is necessarily tentative, and should, where possible, be used as an adjunct to chemical analysis. The possible use of the aluminum and ferric chloride systems in the preparative separation of acid mucopolysaccharides is uncler investigation.

\section{SUMMARY}

A multiple solvent paper chromatography system is described which tentatively identifies most of the known mammalian acid mucopolysaccharides, when these are examined singly. Certain mucopolysaccharides may be identified with reasonable certainty in complex mixtures of such substances. Protein contamination of significant degree does not appear to interfere with these methods. These chromatographic systems appear to be most useful for the rapid, tentative identification of acid mucopolysaccharides where only limited samples are available.

\section{REFERENCES}

1 IF. Meyer, E. Dhvidson, A. Jinier and P. Hofrinan, Biochim. Biophys. Acta, 2 i (1956) 506. $2 \mathrm{~J}$. E. Scotr. Aliphatic Ammonimm Salts in the Assay of Acidic Polysaccharides from Tissues, in D. Grick (Editor), Methods of Biochemical Analysis, Vol. VIII, Interscience, New York, I96o, p. I 45 .

3 S. Schiller, G. A. Slover and A. Dorfinan, J. Biol. Chemi, $236(1961) 983$.

4 A. J. Bollet, M. W. Straydarian and W. F. Simpson, J. Clin. Invest., 36 (1957) 328.

5. N. DiFierrante and C. IRich, J. Lab. Clin. Med., 48 (1956) 49 r.

- Tr. li. Princte and C. W. Castor, Expll. Cell lies., 23 (19Gi) Gis.

7 G. T. KERBY, J. Clin. Invest., 33 (1954) i.los.

8 L. Spotter and W. Marx, Biochim. Biopliys. Acta. 38 (1960) raz.

D E. TR. DAlFeres JR. AND G. S. Berlenson, Federation Proc., 20 (1961) 103. (abstract).

10 IS. Meyde, A. Linker, E. A. Davidson and B. Wetssman, J. Biol. Chem., 205 (r953) 6r1. 11 T. G. Kantor and M. Schubert, J.Am. Chem. Soc., 79 (t957) i 52.

$12 \mathrm{~S}$. IRoseman, D. R. Watson, I. F. Durf and W. D. Robinson, Federation Proc., 14 (195.5) 312 (abstract $878 \mathrm{~A}$ ).

13 iM. B. Mathews, Federation Proc., 20 ( $190 \mathrm{~g}$ ) I $0_{3}$ (abstract).

14 C. W. Castor, R. K. Princte and E. L. Dorstewitz, Lab. Invest., i i (r962) 703. 